\title{
A Rare Cause of Dysphagia: A Case Report
}

\author{
Aroor Rajeshwari · Gangadhara Somayaji • \\ Shetty Deviprasad
}

Received: 19 October 2008/Accepted: 28 March 2009/Published online: 11 April 2011

(C) Association of Otolaryngologists of India 2011

\begin{abstract}
Dysphagia is a common clinical symptom for an ENT surgeon in his clinic, evaluation of which may not be a great challenge. The objective of this article is to report a rare cause of dysphagia which may be difficult to diagnose unless specifically thought of. This is a report of a case of dysphagia secondary to myasthenia gravis seen in a young lady along with tongue fasciculation in an early onset case unlike the ones reported in the literature where the patients are elderly and more often males in the late phase of the disease. At times, dysphagia may be seen secondary to some rare causes and may mislead the doctor. A carefully taken history and methodical clinical examination will avoid unnecessary invasive procedures and delay in the diagnosis.
\end{abstract}

Keywords Dysphagia - Myasthenia gravis - Tongue fasciculation

\section{Introduction}

Even though dysphagia is a common symptom in an ENT outpatient, myasthenia gravis causing this symptom is rare. Myasthenia gravis is an autoimmune condition associated with weakness and fatigability of voluntary muscles [1]. The diagnosis is often missed because the disease is rare and the symptoms are fluctuant. Bulbar manifestation as a presenting symptom is rare and mainly seen in elderly men [1]. This is a report of one such case of early onset myasthenia gravis with bulbar weakness as a presenting symptom.

A. Rajeshwari $(\bowtie) \cdot$ G. Somayaji · S. Deviprasad

Yenepoya Medical College, Mangalore, India

e-mail: rajeshwarisomayaji@yahoo.co.in

\section{Case Report}

A 37 year old lady presented to the ENT outpatient with sudden onset of severe dysphagia of 3 days duration. Dysphagia was for both solids and liquids with history of occasional dribbling of saliva. There was no history suggestive of foreign body impaction and aspiration. On Indirect Laryngoscopic examination, both the vocal cords were normal and there was no mass lesion seen in larynx and hypopharynx. There was no pooling of saliva in both the pyriform fossa. Rest of the ENT examination was also normal. Routine blood and urine investigations were normal. X-ray barium swallow was done with thin barium which showed smooth flow of the barium into the stomach.

Upper GI endoscopy was also normal. Three days after the admission, patient developed intermittent tongue fasciculations, slurred speech and mild ptosis of the left eye. A neurological cause was suspected and the patient was referred to the neurologist. Mean while, to our surprise, the fasciculations and the ptosis improved on the next day. Neurologist suspected myasthenia gravis and nerve conduction study was done which was prolonged (Fig. 1). Acetylcholine receptor antibody binding antibody levels were elevated. The patient was put on tab neostigmine $15 \mathrm{mg}$ three times a day, which resulted in significant improvement in dysphagia. Presently, the patient is asymptomatic after 1 year of follow up.

\section{Discussion}

Myasthenia gravis is an autoimmune disorder of the neuromuscular junction characterized by muscle weakness and fatigue [2]. Due to IgG antibodies directed against acetylcholine receptors at the neuromuscular junction [3]. Annual 
Fig. 1 Chart showing prolonged nerve conduction study

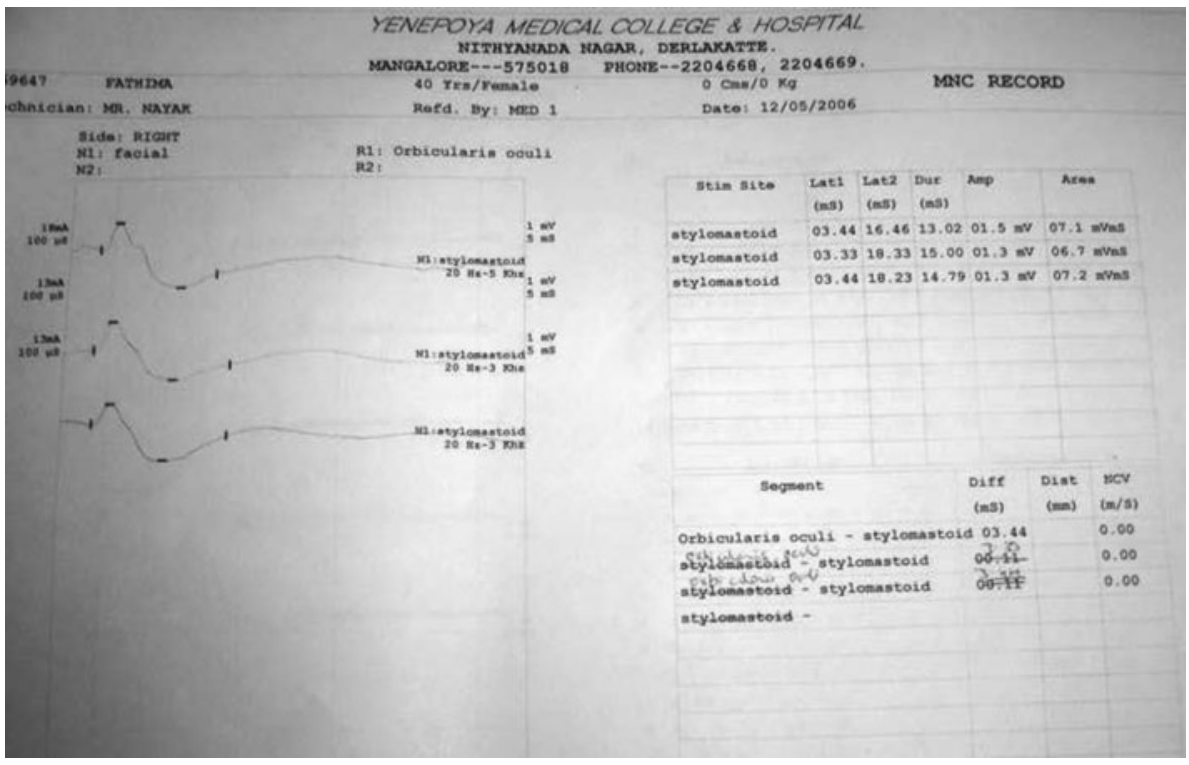

incidence of the disease is widely accepted as 2-4 per million [1]. It is twice as common in females with peak age of diagnosis in the third decade [1]. It is unusual for the patients to present with bulbar weakness and it is the presenting complaint in only $6 \%$ of the patients [1]. Bulbar symptoms as a presentation of the disease are more common in elderly patients, that is, late onset myasthenia gravis [1]. Tongue and masticatory weakness are due to involvement of hypoglossal and trigeminal nerve [1]. Dysphagia could be due to oropharyngeal or oesophageal muscle weakness $[1,2]$. Tongue atrophy has been described in only a minority of the patients and it was thought to present at a later stage in the progression of the disease [1]. After an extensive search in the world literature, only one reported case of myasthenia gravis with tongue muscle fasciculation as a presenting symptom could be found, that too in late onset myasthenia gravis [1].

Myasthenia gravis can be diagnosed by means of serology, nerve conduction studies with single fiber electromyography (SFEMG) or the classical tensilon test [3]. About $85 \%$ of the patients may have antibodies to the acetylcholine receptor and a positive result is diagnostic for myasthenia gravis; unfortunately, the test is not routinely available [3].

\section{Keypoints}

- Myasthenia gravis can present with bulbar symptoms alone.
- Delay in the diagnosis is common because of the lack of recognition.

- Presentation with tongue fasciculation is very rare. Only one such case has been reported in the English literature so far, that too in an elderly patient.

- Our patient is a young lady who presented with dysphagia and bulbar muscle weakness which is rare in early onset myasthenia gravis.

- Myasthenia gravis should be considered in the differential diagnosis of dysphagia in elderly particularly men.

\section{References}

1. Burch J, Warren Gash C, Ingham V et al (2006) Myasthenia gravis, a rare presentation with tongue atrophy and fasciculation. Age Ageing 35(1):87-88

2. Odasso MM (2006) Dysphonia as first symptom of late onset Myasthenia gravis. J Gen Intern Med 21(6):C4-C6

3. McIntyre K, McVeigh-Smock S, Mourad O (2006) An adult patient with new onset dysphagia. CMAJ 175(10):1203 